\title{
ÖKOBILANZWERKSTATT 2016
}

\section{5. + 16. September in Pforzheim}

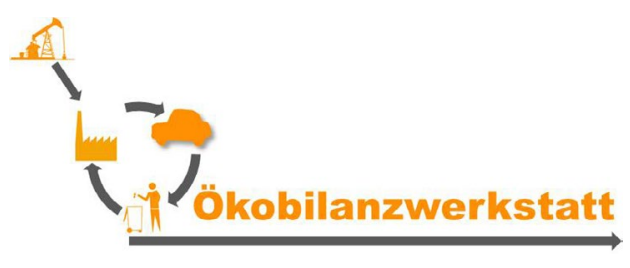

(C) Springer-Verlag Berlin Heidelberg 2016

Die Ökobilanzwerkstatt ist eine Veranstaltung des Netzwerks Lebenszyklusdaten und richtet sich an AbsolventInnen, DoktorandInnen und junge WissenschaftlerInnen, die auf dem Gebiet der Lebenszyklusanalyse forschen oder diese für angewandte Fragestellungen einsetzen. Die jährlich stattfindende Veranstaltung soll nicht nur dazu dienen, das eigene Forschungsthema vorzustellen, sondern den TeilnehmerInnen auch die Möglichkeit bieten, ihre Arbeiten zu diskutieren und Erfahrungen auszutauschen.

Charakteristisch für die Ökobilanzwerkstatt ist die aktive Beteiligung der TeilnehmerInnen. Neben der Möglichkeit die eigene Arbeit im Rahmen eines Vortrags oder Pos- ters zu präsentieren, werden dieses Jahr zusätzlich von den TeilnehmerInnen ausgewählte Themen näher betrachtet, diskutiert und gemeinsam erarbeitet. Des Weiteren werden Experten in Fachvorträgen Einblicke in die entsprechenden Themen geben. Ein gemeinsames Abendprogramm rundet die zweitägige Veranstaltung ab.

Die insgesamt 12. Ökobilanzwerkstatt findet 2016 erneut in Pforzheim statt. Ausrichter ist das Institute for Industrial Ecology (INEC) der Hochschule Pforzheim. Anmeldungen werden bereits entgegengenommen. Das Anmeldeformular und weitere Informationen sind unter http://umwelt. hs-pforzheim.de/oekobilanzwerkstatt/ erhältlich. 